\title{
Tension pneumoperitoneum
}

\author{
P Burdett-Smith, L Jaffey
}

\begin{abstract}
Tension pneumoperitoneum developing in a middle aged asthmatic male during resuscitation after a respiratory arrest is reported. This was associated with bilateral tension pneumothorax and caused severe respiratory embarrassment which was relieved by needle decompression, after decompression of the pneumothoraces. The chest is not the only body cavity that can contain air under tension. (f Accid Emerg Med 1996;13:220-221)
\end{abstract}

Key terms: tension pneumoperitoneum; asthma; resuscitation

\section{Case report}

A 53 year old man with a lifelong history of asthma was brought by paramedics to the accident and emergency (A\&E) department in a state of cardiorespiratory arrest. He had woken that morning with increasingly severe wheeze, which had failed to respond to his usual inhaled therapy. His wife had dialled 999 because he had suddenly become much more breathless, and had developed a stabbing left sided chest pain. The paramedics reported that they had found him on his bed, severely distressed, with gasping respirations. As they were moving him to the ambulance his respiratory rate fell to less than 10 per minute. An oral endotracheal tube was therefore passed (with very little resistance) and $100 \%$ oxygen was given by positive pressure ventilation. Over the course of the next few minutes, he became increasingly difficult to ventilate, his conscious level decreased, and his pulse became impalpable. Chest compression was begun, but this became increasingly difficult. Cardiac monitoring showed sinus tachycardia. Venous access was obtained with moderate difficulty, and adrenaline $1 \mathrm{mg}$ was given intravenously in two bolus doses separated by three minutes. Twenty five minutes after the arrival of the ambulance crew he was transported to the A\&E department.

On arrival he was noted to be deeply cyanosed with massive venous congestion. His chest and abdomen were so rigidly distended that he appeared almost opisthotonic. Attempts at ventilation through the endotracheal tube produced almost no chest movement, and neither did chest compression. Bilateral tension pneumothoraces were diagnosed, and large bore (12 gauge) over-theneedle cannulae were immediately inserted through the second intercostal space in the mid-clavicular line on both sides. Chest compression became noticeably easier, the venous congestion settled, and effective ventilation became possible. Underwater-seal chest drains were promptly inserted, with further improvement, but his abdomen remained grossly distended, with considerable resistance to satisfactory ventilation. A further large bore cannula was therefore inserted percutaneously in the midline to decompress the peritoneal cavity. This resulted in a significant escape of air, accompanied by obvious relief of tension within the abdominal cavity. Ventilatory pressure returned to normal, and cardiac output became sufficient to produce a systolic blood pressure of $110 \mathrm{~mm} \mathrm{Hg}$. Thereafter the cardiovascular signs remained stable, but there were no signs of cerebral activity. $\mathrm{He}$ was admitted to the intensive therapy unit, where, on evidence of the criteria for brainstem death, he was later pronounced dead.

\section{Discussion}

Tension pneumoperitoneum was first reported in $1913^{1}$ when gas forming organisms were thought to be responsible. More recent cases have been associated with recent surgery to the bowel and positive pressure ventilation. Such a case was described in 1981 in a 27 year old male who required operation for a stab wound to the abdomen. ${ }^{2}$ Upon induction of anaesthesia, a tension pneumoperitoneum developed from a perforation of the greater curvature of the stomach, causing high inspiratory pressures which were relieved by emergency laparotomy with the release of a large quantity of air under tension. A similar case was described in a 36 year old man who developed a tension pneumoperitoneum nine days after subtotal colectomy. ${ }^{3}$ In this case aerophagy was thought to be the causative factor, rather than positive pressure ventilation. Three cases of pneumoperitoneum after blunt trauma, with no evidence of bowel perforation, were described in $1979,{ }^{4}$ but none of these was under tension.

Pneumoperitoneum under tension was described following surgery and positive pressure ventilation, ${ }^{5}$ but a case was also described in which the ventilation appeared to be the only cause. In this case, adult respiratory distress syndrome (ARDS) had developed and high inspiratory pressures were needed, leading to barotrauma and the development of pneumothorax. A similar case was described in a 26 year old female who also developed ARDS requiring high ventilatory pressures. ${ }^{6}$ Although she had undergone laparotomy for emergency hysterectomy postpartum, analysis of the aspirated air confirmed the origin from the thorax as opposed to bowel perforation.

Two cases of pneumoperitoneum associated with tension pneumothorax have been 
described, ${ }^{7}$ although the pneumoperitoneum was not under tension in either case. In both, lung disease was present and the patients required mechanical ventilation with high inspiratory pressures. Tension pneumoperitoneum can also develop without positive pressure ventilation from perforation of abdominal viscera. ${ }^{8-10}$

Possible mechanisms for air entering the peritoneal space from the pleural space have been described previously. ${ }^{11}{ }^{12}$ Air may leak through the alveolar walls during high pressure ventilation and thence along the perivascular sheaths to the mediastinum and by the diaphragmatic openings to the retroperitoneal space and the peritoneum. This theory is supported by a recent report of pneumoretroperitoneum in a low birthweight infant associated with tension pneumothorax. ${ }^{13}$ There may alternatively be direct communication between the peritoneal and pleural spaces through diaphragmatic defects, which may be congenital or traumatic. A large increase in the intra-abdominal pressure, caused by tension pneumoperitoneum, as well as raising the diaphragm and causing respiratory embarrassment, can compress the inferior vena cava, causing diminished venous return, reduced cardiac output, and lowered mean arterial blood pressure. This increase in intraabdominal pressure can high enough to compress the aorta. ${ }^{8}$ The clinical signs of respiratory embarrassment, abdominal distension, and possibly cyanosis in a patient with high ventilatory pressures may be an indication of raised intra-abdominal pressure. In an emergency situation, consideration should be given to decompression of the peritoneal cavity by large bore needle, as in the treatment of tension pneumothorax.

Tension pneumoperitoneum occurring in a resuscitation setting, as opposed to that associated with mechanical ventilation on the intensive care unit, has not to our knowledge been described before. Reports confirm that pneumoperitoneum under tension is very rare and appears to require a combination of lung disease and mechanical ventilation with high inspiratory pressures. Most cases of pneumoperitoneum are secondary to anastomatic leakage or previous bowel injury. This may cause a rise in intra-abdominal pressure, especially in the presence of mechanical ventilation.

Tension pneumothorax is a well recognised complication of mechanical ventilation, especially in the presence of pre-existing lung disease. Rapid relief of the raised intrapleural pressure by needle thoracocentesis or tube thoracostomy is life saving. In the case presented, bilateral needle decompression of the chest only partially relieved the respiratory embarrassment. When a needle was inserted into the peritoneal cavity, the ventilatory pressures were markedly reduced.

Patients who develop acute respiratory distress during artificial ventilation should be assessed for signs of air under tension in the chest cavity, but may also have raised intraperitoneal pressure from tension pneumoperitoneum.

1 Falkenburg C. Ein Fall von Gasansammlung in der freien Bauch-Hohle. Dtsch Z Chir 1913;124:130-6.

2 Roberts R, Blake B, Bruggeman G. Tension pneumoperitoneum - a cause of ventilatory obstruction. Anesthesiperitoneum - a cause

3 Higgins J, Halpin D, Midgley A. Tension pneumoperitoneum: a surgical emergency. Br f Hosp Med 1988; peritoneum:

4 Andrew T, Milne D. Pneumoperitoneum associated with pneumothorax or pneumopericardium: a surgica dilemma in the injured patient. Injury 1979;11:65-70.

5 Winer-Muram $H$, Rumbak M, Bain R. Tension pneumoperitoneum as a complication of barotrauma. Crit Care Med 1993;21:941-3.

6 Ralston C, Clutton-Brock TH, Hutton P. Tension pneumoperitoneum. Intensive Care Med 1989;15:532-33.

7 Egan M, Boutros A. Pneumoperitoneum following tension pneumothorax. Report of two cases. Crit Care Med 1975;3:170-2.

8 Olinde A, Carpenter D, Maher J. Tension pneumo-

peritoneum. Arch Surg 1983;118:1347-50. a report of 4 cases. Br $\mathcal{F}$ Surg 1976;63:877-80.

10 Addison N V. Tension pneumoperitoneum. A report on two cases. Br $\mathcal{F}$ Surg 1959;45:411-12.

11 Macklin C. Transport of air along sheaths of pulmonic blood vessels from alveoli to mediastinum. Arch Intern blood vessels from alve

12 Joannides $M$, Tsoulos $G$. The etiology of interstitial and mediastinal emphysema. Arch Surg 1930;21:333.

13 Karlowicz M. Pneumoretroperitoneum and perirenal air associated with tension pneumothorax. Am $\mathcal{f}$ Perinatol 1994;11:63-4.

\section{Accident and Emergency Department, Dundee Royal Infirmary, Barrack Road, Dundee G W Kerr \\ Correspondence to: Dr Gary W Kerr, Orthopaedic Department, Perth Royal Infirmary, PH1 1 NX}

\title{
Dystonic reactions: two case reports
}

\author{
Gary W Kerr
}

\begin{abstract}
Case reports of dystonic reactions to metoclopramide are presented. Dystonic reactions may occur after ingestion of many drugs and should be considered by accident and emergency staff in patients with a suggestive clinical presentation. ( $\mathcal{A}$ Accid Emerg Med 1996;13:221-222)
\end{abstract}

Key terms: dystonic reactions; drug side effects
Dystonic reactions are a well recognised complication of many drugs, ${ }^{12}$ commonly the antipsychotics and antiemetics. Acute dystonias most commonly affect children and young adults, ${ }^{3}$ with the muscles of the head and neck mainly affected. Opisthotonus and torticollis are classical, as are oculogyric crises with painful lateral or vertical deviation of the eyes. Bizarre grimaces, blepharospasm, tongue protrusion, and a subjective feeling of swelling 